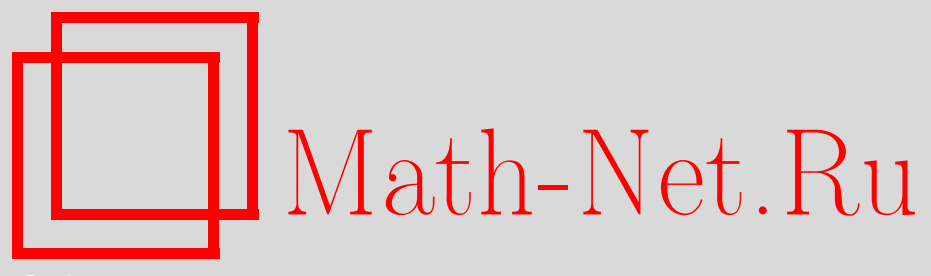

В. Г. Кадышевский, А. С. Сорин, К вопросу об интегрируемой структуре полевой теории открытых суперструн, TMФ, 2006, том 149, номер 3, 381-385

DOI: https://doi.org/10.4213/tmf5529

Использование Общероссийского математического портала Math-Net.Ru подразумевает, что вы прочитали и согласны с пользовательским соглашением http://www . mathnet.ru/rus/agreement

Параметры загрузки:

IP : 54.157 .27 .8

26 апреля 2023 г., $17: 34: 35$

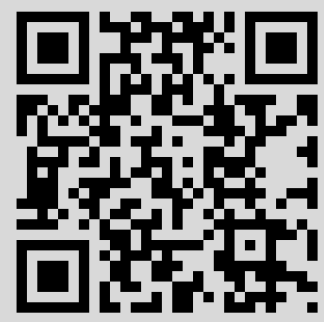




\title{
К ВОПРОСУ ОБ ИНТЕГРИРУЕМОЙ СТРУКТУРЕ ПОЛЕВОЙ ТЕОРИИ ОТКРЫТЫХ СУПЕРСТРУН
}

\begin{abstract}
Найдены асимптотические разложения по "струнной массе" $\mu$ для $\mu$-деформированных Г-функций и коэффициентов Неймана, характеризующих трехструнную вершину в калибровке светового конуса полевой теории открытых суперструн на максимально суперсимметричном $p p$-волновом фоне.
\end{abstract}

Ключевые слова: полевая теория струн, пространство анти-де Ситтера, иерархия Тоды.

Посвящается 80-летию академика Анатолия Алексеевича Логунова

Пять лет назад в работе [1], посвященной 75-летию академика Анатолия Алексеевича Логунова, мы рассмотрели квазиклассический предел суперсимметричной решеточной иерархии Тоды. За прошедшие годы мы неоднократно возвращались к этому кругу идей [2]-[4] и нашли неожиданные применения их в ряде разделов современной теоретической и математической физики. Выяснилось, в частности, что квазиклассический (бездисперсионный) предел решеточной иерархии Тоды связан с интегрируемой структурой, лежащей в основе полевой теории открытых (супер)струн на плоском геометрическом фоне [3], [4]. Было установлено, что коэффициенты трехструнной вершины полевой теории открытых (супер)струн в ковариантной калибровке и калибровке светового конуса удовлетворяют уравнениям Хироты бездисперсионной иерархии Тоды. Это позволило вычислить корреляторы соответствующей квантовой системы, коэффициенты Неймана которой являются двухточечными функциями.

Рассмотрение более общего случая, когда фоном служит пространство анти-де Ситтера $A d S_{5} \times S^{5}$, оказавшееся в последние годы в фокусе внимания в связи с гипотезой $\mathrm{AdS} / \mathrm{CFT-соответствия,} \mathrm{расширило} \mathrm{класс} \mathrm{специальных} \mathrm{функций} \mathrm{за} \mathrm{счет}$ привлечения так называемой $\mu$-деформированной Г-функции [5], [6]. В работе [4] была высказана гипотеза, что интегрируемой структурой полевой теории открытых суперструн на плосковолновом пределе пространства $A d S_{5} \times S^{5}$, максимально

* Объединенный институт ядерных исследований, Дубна, Московская область, Россия. E-mail: kadyshev@jinr.ru, sorin@theor.jinr.ru 
суперсимметричном $p p$-волновом фоне, является полная (дисперсная) решеточная иерархия Тоды, в которой шагом решетки служит "струнная масса" $\mu$ - обратный радиус пространства анти-де Ситтера. Это предположение проверялось путем разложения по $\mu$ с точностью до второго порядка включительно, было показано, что с этой точностью коэффициенты трехструнной вершины в калибровке светового конуса действительно удовлетворяют уравнениям Хироты полной (дисперсной) решеточной иерархии Тоды.

Однако до последнего времени некоторые принципиальные проблемы в данном подходе оставались нерешенными. В частности, для $\mu$-деформированных Г-функции и коэффициентов Неймана, характеризующих трехструнную вершину полевой теории открытых суперструн на максимально суперсимметричном $p p$-волновом фоне, нужно было построить асимптотические разложения по $\mu$, необходимые для проверки вышеупомянутой гипотезы в следующих порядках разложения по "струнной массе". В настоящей статье, которая будет опубликована в канун 80-летия А. А. Логунова, приведено решение указанной проблемы. Пользуясь случаем, мы хотим пожелать юбиляру доброго здоровья и новых творческих достижений.

Нашей отправной точкой будут $\mu$-деформированные коэффициенты Неймана [6]

$$
\begin{gathered}
\mathcal{N}_{m n}^{r s}=-\frac{\alpha m n}{\alpha_{r} \alpha_{s}\left(\alpha_{s} \omega_{r, m, \mu}+\alpha_{r} \omega_{s, n, \mu}\right)} \mathcal{N}_{m}^{r} \mathcal{N}_{n}^{s}, \\
\mathcal{N}_{m}^{r}=\sqrt{\frac{\omega_{r, m, \mu}}{m}}\left(1+\frac{\alpha_{r}}{\tau_{0}} \frac{\mu}{\omega_{r, m, \mu}}\right) \frac{\beta_{r-1} \Gamma_{\mu}^{(r+1)}\left(-\beta_{r} m\right)}{m^{2} \Gamma_{\mu}^{(r)}(m) \Gamma_{\mu}^{(r-1)}\left(-\beta_{r} m-m\right)} e^{\frac{\tau_{0}}{\alpha_{r}} \omega_{r, m, \mu}},
\end{gathered}
$$

которые выражаются через введенную в [5], [6] $\mu$-деформированную Г-функцию

$$
\begin{aligned}
\Gamma_{\mu}^{(r)}(z) & :=\frac{e^{-\gamma \omega_{r, z, \mu}}}{z} \prod_{n=1}^{\infty}\left(\frac{n}{\omega_{r, n, \mu}+\omega_{r, z, \mu}} e^{\frac{\omega_{r, z, \mu}}{n}}\right), \\
\Gamma_{\mu}^{(r)}(z) \Gamma_{\mu}^{(r)}(-z) & =-\frac{\pi}{z \sin (\pi z)}, \quad \Gamma_{\mu=0}^{(r)}(z)=\Gamma(z) .
\end{aligned}
$$

Здесь по сравнению с [6] мы изменили масштаб $\mu\left(\mu \rightarrow \mu / \tau_{0}\right)$, а также несколько модифицировали определение Г-функции $\left(\Gamma_{\mu}^{(r)}(z) \rightarrow \Gamma_{\mu}^{(r)}\left(z / \alpha_{r}\right)\right)$. Мы используем следующие обозначения: $\gamma$ - постоянная Эйлера; натуральные числа $r, s=1,2,3$ по модулю $3 ; m, n=1,2, \ldots$,

$$
\omega_{r, z, \mu}:=\operatorname{sgn}(z) \sqrt{z^{2}+\left(\frac{\alpha_{r} \mu}{\tau_{0}}\right)^{2}}
$$


$\alpha, \alpha_{r}, \tau_{0}, \beta_{r}, \beta, \mu$-вещественные параметры, удовлетворяющие условиям

$$
\begin{aligned}
\alpha & :=\alpha_{1} \alpha_{2} \alpha_{3}, \quad \tau_{0}:=\sum_{r=1}^{3} \alpha_{r} \ln \left|\alpha_{r}\right|, \quad \sum_{r=1}^{3} \alpha_{r}=0, \\
\beta_{r} & :=\frac{\alpha_{r+1}}{\alpha_{r}}, \quad \beta_{1} \beta_{2} \beta_{3}=1, \quad \beta_{r}\left(\beta_{r+1}+1\right)=-1, \\
\beta_{1} & :=\beta, \quad \beta_{2}=-\frac{\beta+1}{\beta}, \quad \beta_{3}=-\frac{1}{\beta+1}, \\
e^{\frac{\tau_{0}}{\alpha_{1}}} & =\frac{|\beta|^{\beta}}{|\beta+1|^{\beta+1}}, \quad e^{\frac{\tau_{0}}{\alpha_{2}}}=\frac{|\beta|}{|\beta+1|^{\frac{\beta+1}{\beta}}}, \quad e^{\frac{\tau_{0}}{\alpha_{3}}}=\frac{|\beta+1|}{|\beta|^{\frac{\beta}{\beta+1}}} .
\end{aligned}
$$

Отметим, что $\mu$-деформированные коэффициенты Неймана (1), (2) являются однородными функциями параметров $\alpha_{r}$, поэтому в действительности они зависят только от двух вещественных параметров $\beta$ и $\mu$.

Нашей ближайшей задачей будет получение асимптотического разложения $\Gamma_{\mu}^{(r)}(z)$ по $\mu$ при $|\mu|<1$, для того чтобы затем вывести соответствующие разложения для $\mathcal{N}_{m n}^{r s}$ и $\mathcal{N}_{m}^{r}$. Представление $\Gamma_{\mu}^{(r)}(z)$ в виде бесконечного произведения (3) неудобно для этой цели. По этой причине мы найдем более подходящий вид $\Gamma_{\mu}^{(r)}(z)$.

Из определения (3) вытекает очевидное соотношение

$$
\frac{\partial}{\partial \mu} \ln \Gamma_{\mu}^{(r)}(z)=-\left(\frac{\alpha_{r}}{\tau_{0}}\right)^{2} \frac{\mu}{\omega_{r, z, \mu}}\left(\gamma+\sum_{n=1}^{\infty}\left(\frac{1}{\omega_{r, n, \mu}}-\frac{1}{n}\right)\right) .
$$

Вычисляя сумму и интегрируя с учетом граничного условия, находим $\left(J_{0}(x)\right.$ - функция Бесселя нулевого порядка)

$$
\begin{aligned}
\Gamma_{\mu}^{(r)}(z)= & \Gamma(z) \exp \left\{-\left(\frac{\alpha_{r}}{\tau_{0}}\right)^{2} \int_{0}^{\mu} d x \frac{x}{\omega_{r, z, x}}\left[\gamma+\sum_{n=1}^{\infty}\left(\frac{1}{\omega_{r, n, x}}-\frac{1}{n}\right)\right]\right\} \equiv \\
\equiv & \Gamma(z) \exp \left\{-\left(\frac{\alpha_{r}}{\tau_{0}}\right)^{2} \int_{0}^{\mu} d x \frac{x}{\omega_{r, z, x}} \times\right. \\
\times & {\left.\left[\gamma+\int_{0}^{\infty} \frac{d t}{e^{t}-1}\left(J_{0}\left(\frac{\alpha_{r}}{\tau_{0}} x t\right)-J_{0}(0)\right)\right]\right\} . }
\end{aligned}
$$

Это выражение удобно для получения асимптотических разложений по $\mu$ как при $|\mu| \ll 1$, так и при $|\mu| \gg 1$.

Ограничимся здесь только случаем $|\mu|<1$. Подставляя

$$
J_{0}(x)=\sum_{k=0}^{\infty} \frac{(-1)^{k}}{(k !)^{2}}\left(\frac{x}{2}\right)^{2 k}
$$

в (11) и интегрируя по $t$, получаем

$$
\begin{aligned}
\Gamma_{\mu}^{(r)}(z)=\Gamma( & z) \exp \left\{-\left(\frac{\alpha_{r}}{\tau_{0}}\right)^{2} \int_{0}^{\mu} d x \frac{x}{\omega_{r, z, x}} \times\right. \\
\times & {\left.\left[\gamma+\sum_{k=1}^{\infty}(-1)^{k} \frac{(2 k) !}{(k !)^{2}} \zeta(2 k+1)\left(\frac{\alpha_{r}}{2 \tau_{0}} x\right)^{2 k}\right]\right\}, }
\end{aligned}
$$


где $\zeta(k)$ - дзета-функция Римана. Используя разложение $\left(\omega_{r, z, x}\right)^{-1}$ в степенной ряд по $x^{2}$,

$$
\frac{1}{\omega_{r, z, x}}=\frac{1}{z} \sum_{l=0}^{\infty} \frac{\Gamma\left(\frac{1}{2}\right)}{l ! \Gamma\left(\frac{1}{2}-l\right)}\left(\frac{\alpha_{r} x}{\tau_{0} z}\right)^{2 l}
$$

и интегрируя по $x$, мы можем без труда получить разложение $\Gamma_{\mu}^{(r)}(z)$ в степенной ряд по $\mu$.

Подставляя (11)-(13) в (2) и принимая во внимание соотношения

$$
\omega_{r+1,-\beta_{r} m, \mu}=-\beta_{r} \omega_{r, m, \mu}, \quad \omega_{r-1,-\beta_{r} m-m, \mu}=\frac{1}{\beta_{r-1}} \omega_{r, m, \mu},
$$

получаем следующее представление для $\mathcal{N}_{m}^{r}$ :

$$
\mathcal{N}_{m}^{r}=N_{m}^{r} \sqrt{\frac{\omega_{r, m, \mu}}{m}}\left(1+\frac{\alpha_{r}}{\tau_{0}} \frac{\mu}{\omega_{r, m, \mu}}\right) e^{\phi_{r, m, \mu}},
$$

где

$$
\begin{aligned}
\phi_{r, m, \mu}:= & \frac{\tau_{0}}{\alpha_{r}}\left(\omega_{r, m, \mu}-m\right)+\frac{\alpha_{r}}{\tau_{0}} \int_{0}^{\mu} d x \frac{x}{\omega_{r, m, x}} \sum_{n=1}^{\infty} \sum_{s=1}^{3} \frac{\alpha_{s}}{\tau_{0} \omega_{s, n, x}} \equiv \\
\equiv & \frac{\tau_{0}}{\alpha_{r}}\left(\omega_{r, m, \mu}-m\right)+\frac{\alpha_{r}}{\tau_{0}} \int_{0}^{\mu} d x \frac{x}{\omega_{r, m, x}} \int_{0}^{\infty} \frac{d t}{e^{t}-1} \sum_{s=1}^{3} \frac{\alpha_{s}}{\tau_{0}} J_{0}\left(\frac{\alpha_{s}}{\tau_{0}} x t\right) \equiv \\
\equiv & \frac{\tau_{0}}{\alpha_{r}}\left(\omega_{r, m, \mu}-m\right)+2 \frac{\alpha_{r}}{\tau_{0}} \sum_{k=1}^{\infty}(-1)^{k} \frac{(2 k) !}{(k !)^{2}} \zeta(2 k+1) \times \\
& \quad \times \sum_{s=1}^{3}\left(\frac{\alpha_{s}}{2 \tau_{0}}\right)^{2 k+1} \int_{0}^{\mu} d x \frac{x^{2 k+1}}{\omega_{r, m, x}} .
\end{aligned}
$$

Отсюда после некоторых преобразований можно получить разложение в степенной ряд по $\mu$ для $\mu$-деформированных коэффициентов Неймана $\mathcal{N}_{m}^{r}$ : имеем

$$
\begin{aligned}
\phi_{r, m, \mu} & =\frac{\tau_{0}}{\alpha_{r}}\left(\omega_{r, m, \mu}-m\right)+ \\
& +\sum_{k=1}^{\infty} \sum_{l=0}^{\infty}(-1)^{k} \frac{(2 k) ! \zeta(2 k+1) \Gamma\left(\frac{1}{2}\right)}{(k !)^{2}(l+k+1) l ! \Gamma\left(\frac{1}{2}-l\right)}\left(\frac{\alpha_{r}}{\tau_{0} m}\right)^{2 l+1} \sum_{s=1}^{3}\left(\frac{\alpha_{s}}{2 \tau_{0}}\right)^{2 k+1} \mu^{2(l+k+1)} .
\end{aligned}
$$

Представление в виде степенного ряда по $\mu$ оставшихся сомножителей в (16) и (1) может быть получено без труда. Обратим внимание на то, что члены суммы в (17) дают вклад в $\mathcal{N}_{m}^{r}$, начиная лишь с порядка $\mu^{4}$.

Таким образом, нами построены асимптотические разложения по "струнной массе" $\mu$ для $\mu$-деформированных Г-функций (3) и коэффициентов Неймана $\mathcal{N}_{m n}^{r s}(1)$ и $\mathcal{N}_{m}^{r}(2)$, характеризующих трехструнную вершину в калибровке светового конуса полевой теории открытых суперструн на максимально суперсимметричном $p p$ волновом фоне. Ясно, что проверка уравнений Хироты в порядке $\mu^{4}$ может иметь 
существенное значение для обсуждавшейся во введении гипотезы о том, что интегрируемой структурой полевой теории открытых суперструн на максимально суперсимметричном $p p$-волновом фоне является решеточная (дисперсная) иерархия Тоды с шагом решетки, определяемым “струнной массой” $\mu$. Мы надеемся вернуться к этой проблеме в последующих публикациях.

Благодарности. Авторы выражают благодарность Л. Боноре за многочисленные полезные обсуждения и сотрудничество. Данная работа была частично поддержана РФФИ (грант № 06-01-00627-а), РФФИ-ННИО (грант № 06-02-04012-а), DFG (грант № 436 RUS 113/669-3), HATO (грант PST.GLG.980302), Программой поддержки ведущих научных школ (грант № НШ-5332.2006.2) и Программой Гейзенберг-Ландау.

\section{Список литературы}

[1] В. Г. Кадышевский, А. С. Сорин, ТМФ, 132:2 (2002), 222; nlin.SI/0206044.

[2] V. G. Kadyshevsky, A. S. Sorin, "Supersymmetric Toda lattice hierarchies", Integrable Hierarchies and Modern Physical Theories, eds. H. Aratyn, A. S. Sorin, Kluwer Acad. Publ., Dordrecht/Boston/London, 2001, 289; nlin.SI/0011009; V. V. Gribanov, V. G. Kadyshevsky, A.S. Sorin, Discrete Dyn. Nat. Soc., 2004:1 (2004), 113; nlin.SI/0311030; Czech. J. Phys., 54 (2004), 1289; В.В. Грибанов, В.Г. Кадышевский, А.С. Сорин, TMФ, 146 (2006), 90; nlin.SI/0505039; V. V. Gribanov, V. G. Kadyshevsky, A. S. Sorin, Nucl. Phys. B, 727 (2005), 564; nlin.SI/0506041.

[3] L. Bonora, A. S. Sorin, Phys. Lett. B, 553 (2003), 317; hep-th/0211283.

[4] L. Bonora, R. J. Scherer Santos, A. S. Sorin, D. D. Tolla, Class. Quantum. Grav., 23 (2006), 799; hep-th/0511006.

[5] J. Lucietti, S. Schafer-Nameki, A. Sinha, Phys. Rev. D, 69 (2004), 086005; hep-th/0311231; 70 (2004), 026005; hep-th/0402185.

[6] J. Lucietti, Phys. Lett. B, 598 (2004), 285; hep-th/0407035.

Поступила в редакцию 12.07.2006

3 Теоретическая и математическая физика, т. 149, № 3, 2006 г. 